\title{
Incorporating air density into a Gaussian Process wind turbine power curve model for improving fitting accuracy
}

\author{
Ravi Kumar Pandit, David Infield and James Carroll \\ University of Strathclyde, Glasgow \\ United Kingdom (UK)
}

\begin{abstract}
A power curve conventionally represents the relationship between hub height wind speed and wind turbine power output. Power curves facilitate the prediction of power production at a site and are also useful in identifying the significant changes in turbine performance which can be vital for condition monitoring. However, their accuracy is significantly influenced by changes in air density, mainly when the turbine is operating below rated power. A Gaussian Process (GP) is a non-parametric machine learning approach useful for power curve fitting. Critical analysis of temperature correction is essential for improving the accuracy of wind turbine power curves. The conventional approach is to correct the data for air density before it is binned to provide a power curve, as described in the IEC standard.

In this paper, four different possible approaches of air density correction and its effect on GP power curve fitting model accuracy are explored to identify whether the traditional IEC approach used for air density correction is most effective when estimating power curves using a GP. Finding the most accurate air density compensation approach is necessary to minimize GP model uncertainty. keywords: air density, Gaussian Process, power curve, power performance, wind turbine.
\end{abstract}




\section{Abbreviations}

$\begin{array}{ll}\text { CIs } & \text { - Confidence intervals } \\ \text { HAWTs } & \text { - Horizontal-axis wind turbines } \\ \text { GP } & - \text { Gaussian Process } \\ \mathrm{K} & - \text { The general covariance matrix } \\ \mathrm{k}_{\mathrm{SE}} & - \text { Squared exponential covariance function } \\ \text { MAE } & - \text { Mean absolute error } \\ \text { MSE } & - \text { Mean square error } \\ \text { MAPE } & - \text { Mean absolute percentage error } \\ \text { QQ Plot } & - \text { Quantile Quantile plot } \\ \text { RMSE } & - \text { Root mean square error } \\ R^{2} & - \text { Coefficient of determination } \\ \text { SCADA } & - \text { Supervisory control and data acquisition } \\ \text { WTs } & - \text { Wind Turbines }\end{array}$

\section{Introduction}

Wind turbine (WT) power curves are essential for assessing whether individual turbines meet the performance expectations described by original equipment manufacturers (OEMs). They are also central to energy yield estimation. More recently power curves have been seen to have the potential for wind turbine condition monitoring, see for example [1,2]. X. Jia et al. [3] have proposed a performance assessment algorithm based on a similarity metric for the machine performance curve in which the health of the WT is validated by performing principal component analysis of the quasi-linear region of the power curve. With the help of a power curve, performance deterioration associated with faults on an individual wind turbine can be identified, see [4,5].

Various parametric and non-parametric approaches, [6,7], have been used for power curve modeling in the past; the results indicate that in general nonparametric models perform better than parametric models. For example, the author of [8] argued that a non-parametric model is suitable for dealing with large datasets and that it can incorporate the effects of different parameters other 
than wind speed on power curves more easily than parametric models. The work presented in [2] claims that power curve modeling using an artificial neural network (ANN) gives better accuracy than a parametric model, whilst [9] concludes that cluster center fuzzy logic modeling can give an RMSE (root mean square error) value that is much lower than for a least squares fitted polynomial (i.e., parametric) model. Despite this, the author of [6] identifies that a modified hyperbolic tangent (MHTan) model with backtracking search algorithm (BSA) gives an enhanced parametric model for power curve modeling that yields better accuracy than a fuzzy model. Advanced algorithms like the Genetic algorithm (GA) and particle swarm optimization are popular for fitting parametric models, while neural networks, k nearest neighbor clustering $(\mathrm{kNN})$, fuzzy c-mean clustering and machine learning processes, such as Gaussian Processes, are now finding application for nonparametric approaches to engineering problems, as summarized in $[8,10]$.

Power generation from a wind turbine operating below rated power is in theory proportional to air density. Air density is straightforwardly calculated from the measurement of ambient air temperature and pressure. The IEC standard (61400-12-1), [11], references the power curve, known as the standard power curve, to a given standard reference air density. Wind turbine manufacturers supply these standard power curves, and these provide the commercial basis of sales contracts. Accurate wind power forecasting requires accurate power curves and ensures low wind curtailment and underpins efficient wind project planning for construction and operation. While constructing a forecasting model, particular attention should be paid to the uncertainty associated with the forecasts. For example, the author of [12] proposed a nonparametric system to forecast wind speed uncertainty based on recurrence analysis techniques. Based on chaos theory, this approach models the inherent dynamic characteristics of wind speed which assists in exploring the modeling of uncertainty. Also, the same authors, [12], constructed a frequency domain model to represent uncertainty and results show that this is a more effective and robust than the benchmark models.

Energy produced by a wind turbine operating below rated power is stochastic, reflecting the nature of wind, and hence accurate estimation of any change in conditions is necessary, [13]. Particular attention should be paid to the air density since it significantly affects wind power generation and its accuracy. For example, the BARANI company, [14], concludes that weather influences air density significantly and air density can impact wind energy income generation by up to $10 \%$. Unless compensated for, air density changes will add considerable uncertainty to 
estimates of long-term energy yield from wind turbines. Air density depends on the specific wind farm location and most notably on site elevation and ambient temperature, as demonstrated by [15]. Zahariea and Husaru, [16], confirmed that air density correction (via temperature and pressure) should be implemented when undertaking wind resource assessment and for estimating the performance curve of a wind turbine. The author of [17] has shown that assuming air density to be constant reduces the accuracy of power generation estimates, and so it is advisable to select air density based on the actual wind farm on-site conditions. Here authors used two wind generators of rated values of $7.5 \mathrm{~kW}$ (small-scale) and $850 \mathrm{~kW}$ (medium-scale) to analyze the impact of air density on wind generator efficiency. Jung and Kwon, [18], proposed an artificial neural network (ANN) based on error functions to estimate the annual energy production of WTs and results confirmed its accuracy is much better than for conventional ANNs. The authors of [18] utilized three conventional ANN models based on input selections, and these are: a) ANN1 : just wind speed at the reference site used as input; b) ANN2: both wind speeds and directions used as inputs at the reference sites and c) ANN3: used both parameters of ANN2 however in different ways (rather than using the speed and angle, it used $x$ - and $y$-components of the wind velocity vector). In Ref. [18], authors proposed two ANN models; ANN4 and ANN5 in which the inverse of the frequency of the wind speed and power performance curve were used respectively. Both proposed models used the different types of the parameter to calculate the weight applied to each set of training data $\left(C_{k}\right)$. In contrast, the authors of [18] did exclude air density variations from their proposed models and argued that its impact in contrast with wind speed variation was limited since energy production is proportional to air density but to the cube of the wind speed. This may be true, but does not support the exclusion of air density from energy yield modelling. Hau, [19], has highlighted that the difference between air densities recorded at sites with a difference in height of hundreds of meters can be such to significantly influence the power performance of a turbine, and thus should not be ignored. Chi Yan and Cristina L. Archer, [20], carried out an investigation on the effect of compressibility on the performance or large horizontal-axis wind turbines (HAWTs). As part of this study, authors found that treating air density as constant caused a direct change in power estimation. The work showed that relating compressibility to changing air density slightly degraded the turbine efficiency, confirming the relationship between air density and turbine performance. Liu and Liu, [21], found a significant deviation from standard air density in 
inland regions of China that are located at a substantial height above sea level; this is further confirm the importance of air density in estimating the power output of a turbine.

A powerful and compelling curve fitting procedure known as a Gaussian Processes (GP) is a very general stochastic non-linear model. Due to improvements in desktop computing these models are now widely available and feasible for wind turbine power curve fitting. GP power curve fitting comes with intrinsic confidence intervals (that reflect the standard deviation of the model), and this has proved valuable for uncertainty assessment and fault detection analysis, [22]. GPs are also useful in estimating the power load probability density; for example, in [23] a Gaussian Process model based on quantile regression has been used for short-term probabilistic load forecasting which is useful in application areas like grid management and power dispatching. Accurate wind power estimation is vital for the safe and cost-effective use of wind energy, and here GPs have also started to find application; for example,[24], where a GP and a numerical weather prediction (NWP) model were used for power forecasting and found to give $9 \%$ to $14 \%$ improvement in forecasting accuracy over an artificial neural network model. Network integration costs associated with wind power can be significant but substantially reduced by accurate forecasting, and a practical GP application to this is demonstrated in [25].

Following the IEC Standard, wind turbine power curves are calculated using a data reduction technique known as binning. Air density correction is carried out before binning. However, binning is not necessarily the most effective way to generate a power curve from wind speed and wind turbine power data. As discussed above, many approaches have been proposed that include air density in parametric and non-parametric curve fitting approaches; further examples are [26] and [27]. In Ref. [28], a comparative analysis of a binned power curve and fitted GP power curve has been undertaken for the section of the power curve where the data variation is highest, i.e., on the rising section of the power curve. By comparing a binned power curve with the Gaussian Process power curve, it is found that the latter is more accurate over this portion of the power curve. According to the IEC standard, as mentioned above, air density correction is required prior to binning. However, there is uncertainty as to whether this approach will also give the most accurate results when using a GP for curve fitting. An important observation regarding air density correction was found in [29], where two different windfarm datasets were used in a GP model. For one of these data sets, the model accuracy marginally improved by avoiding the standard air density pre-correction. In the present work, data sets with significant air density variations have 
been explored to confirm the tentative conclusions of [29]. A suitable air density approach selection for GP models not only can improve the power curve fitting but is important for constructing robust GP power curve models for anomaly detection.

Improving yields from wind farms is vital to operators, and consequently, the need for accurate power curve modeling is increasing. As described above, power performance is affected by air density, and thus appropriate investigation needs to be undertaken to assess the various conceivable air density compensation approaches and their effect on GP power curve modeling. In this paper different kinds of air density compensation approaches are explored (including the IEC standard approach) within a GP model and their impact on power curve accuracy is assessed. This will lead to an improved air density compensation approach that will prove valuable for the construction of a robust GP algorithm for anomaly detection and other purposes. This the motivation for the work presented here.

To make a definitive assessment, datasets from turbines located in extremely low and hightemperature regions will be used. Since temperature is the most noteworthy factor influencing air density, these datasets have air density values that are very far from the IEC standard air density, and this is key to understanding its role in GP power curve models. As mentioned above, reference [29] hinted that the standard approach to air density correction might not be optimal. The fundamental role this paper is to demonstrate this definitively for the first time and highlight the preferred approach. It is considered that this has significant implications for all applications of wind turbine power curves and specifically their utilization in wind turbine condition monitoring. The outline of this paper is as follows: Section 1 is the introduction. Section 2 describes the different types of air density compensation schemes. Section 3 describes SCADA data sets and its pre-processing. Section 4 proposes a Gaussian Process model for wind turbine power curve modelling and the inclusion of air density. Section 5 describes the comparative analysis of GP models with different air density compensation approaches using performance error metrics; and Section 6 concludes the paper with an outline of intended future work.

\section{Air density compensation approaches}

The IEC standard acknowledges that pressure, temperature, and humidity at the wind farm site affects the air density and so power generation. Of these parameters, temperature has the greatest impact of on air density and compensating for it has resulted in improved power curves. In this research, the impact of temperature and the different ways to compensate for it are assessed with 
a view to reducing the prediction error and improving GP model fitting accuracy; especially in dealing with the uncertainty of the model.

The power produced by the turbine relies upon the weather due to associated air density variations. For a given wind speed, a turbine can produce notably more power in cold weather (due to higher air density) as compared to summer. Highlighting that correct air density adjustment is key to accurate power measurement and forecasting. In power curve analysis, a standard value for air density is used to reflect a typical average air temperature adjusted to sea level.

Following the IEC standard (61400-12-1), air density correction needs to applied to a pitch regulated wind turbine in which a corrected wind speed $V_{C}$ is calculated using equations (1) and (2) as shown below,

$$
\begin{aligned}
& \rho=1.225\left[\frac{288.15}{\mathrm{~T}}\right]\left[\frac{\mathrm{B}}{1013.3}\right] \\
\text { and, } \quad & \mathrm{V}_{\mathrm{C}}=\mathrm{V}_{\mathrm{M}}\left[\frac{\rho}{1.225}\right]^{\frac{1}{3}}
\end{aligned}
$$

where, $V_{C}$ and $V_{M}$ are the corrected and measured wind speed in $\mathrm{m} / \mathrm{sec}$ and the corrected air density is calculated by equation (2) where B is atmospheric pressure in mbar, and $\mathrm{T}$ the temperature in Kelvin in which 10-minute average values obtained from SCADA data are used. The corrected wind speed $\left(V_{C}\right)$ from equation (3) is then used to calculate the power curve, normally by binning. IEC standards suggest two methods for air density correction can be applied to the power curve depending upon the power control system (i.e., pitch or stall regulated). SCADA datasets used in this paper are from pitch regulated wind turbines (WTs). Hence power output is obtained by correcting wind speed as described above.

The air density $\rho$ is related to temperature by the gas law $\rho=\frac{\mathrm{p}}{(\mathrm{R} . \mathrm{T})}$; where $\mathrm{p}$ is absolute atmospheric pressure, $\mathrm{R}$ the gas constant and $\mathrm{T}$ is the environmental temperature in Kelvin. The pressure $\mathrm{p}$ changes with altitude and can thus vary noticeably according to the location of the wind turbine. The environmental temperature significantly affects the air density and hence power capture of a turbine. Because of the significant influence of temperature on air density, air density correction is sometimes referred as temperature correction.

In this paper, four different approaches have been used to compensate (or not) for air density effects when using a GP rather than binning to identify the power curve. These are: a) no pre- 
correction and air density not included in the GP model b) no pre-correction but with air density included within the GP model c) pre-correction applied but without air density in the GP model, and finally d) with pre-correction and air density included with GP model. These four approaches are assessed for their impact on the accuracy of the GP power curve model through the confidence limits associated with the fit.

Flow chart 1 summarizes the proposed research methodology in which extreme SCADA datasets of WTs are first filtered and then used to train the GP models. While doing so, four different proposed air density compensation approaches are incorporated into the GP models in order to analyze their impact on GP model accuracy and uncertainty. Confidence intervals, performance error metrics and calculated residuals are used to assess the impact of these four air density compensation schemes on the GP models.

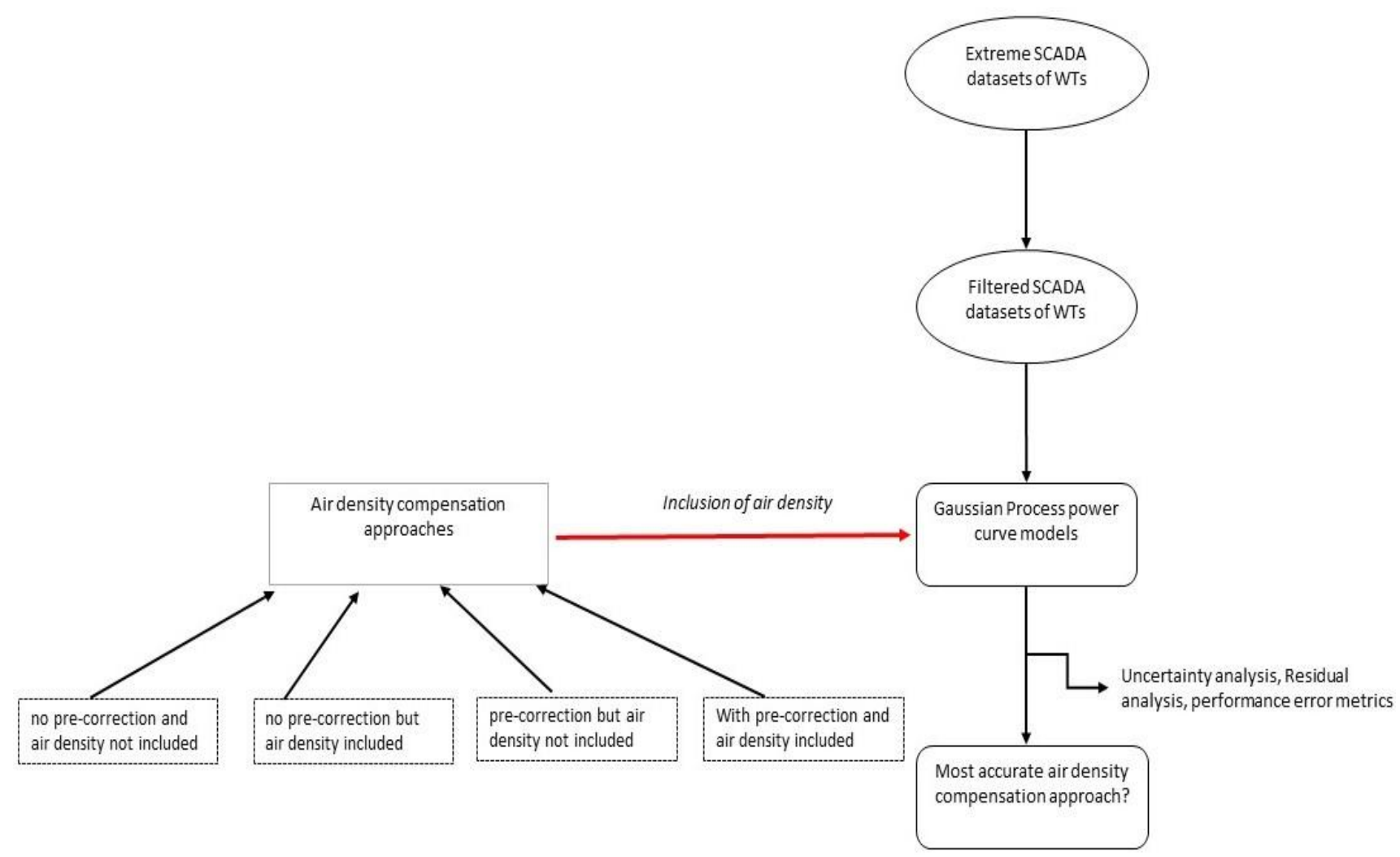

Flow chart 1: Flow chart of air density compensation approaches and Gaussian Process models 


\section{Wind farm description and data preprocessing}

The application of real operational data from wind turbines is a powerful technique for demonstrating the effectiveness of the GP algorithms. Continuous health monitoring can be undertaken efficiently using SCADA data which provides valuable information in regards to load history and operations of individual wind turbines and provides an efficient and cost-effective way to monitor wind turbines for early warning of failures and related performance issues. All the SCADA data used in this paper comprises 10-minute averages with maximum, minimum, standard deviation and an entire month of operational data from two sites used in this study.

According to the IEC standard (61400-12-1) guidelines, the air density correction shall be applied when the site density differs from the standard value $\left(1.225 \mathrm{~kg} / \mathrm{m}^{3}\right)$ by more than $0.05 \mathrm{~kg} / \mathrm{m}^{3}$. In order to investigate the impact of temperature, and also comply with this IEC requirement, two wind turbine datasets; one located in southern Europe (containing extreme high temperatures) and another in northern Europe (containing extreme low temperatures) are used. For the sake of convenience, the two sites designated as follows:

$$
\text { A = northern Europe site; } \quad \text { B = southern Europe site. }
$$

The datasets used are from the year 2010 for which February and August SCADA data from sites $\mathrm{A}$ and $\mathrm{B}$ record extreme average monthly temperatures of $-5.277^{\circ} \mathrm{C}$ at $\mathrm{A}$ and $29.779^{\circ} \mathrm{C}$ at $\mathrm{B}$. For these two particular months the air density differs by -0.102 and 0.061 from the IEC standard value for sites A and B respectively as shown in table 1. Thus both these datasets conform to the IEC standards guideline described above for making air density correction.

\begin{tabular}{|c|c|c|c|c|c|}
\hline WTs & $\begin{array}{l}\text { SCADA datasets } \\
\text { time period }\end{array}$ & $\begin{array}{l}\text { Total number of } \\
\text { data points }\end{array}$ & $\begin{array}{c}\text { Average monthly } \\
\text { temperature } \\
\left({ }^{\circ} \mathrm{C}\right)\end{array}$ & $\begin{array}{c}\text { Standard } \\
\text { density } \\
\left(\mathrm{kg} / \mathrm{m}^{3}\right)\end{array}$ & $\begin{array}{c}\text { Mean absolute } \\
\text { difference } \\
\left(\boldsymbol{k g} / \mathrm{m}^{3}\right)\end{array}$ \\
\hline A & $\begin{array}{l}1 / 02 / 2010- \\
28 / 02 / 2010\end{array}$ & 4032 & -5.2775 & 1.225 & -0.102 \\
\hline B & $\begin{array}{l}1 / 08 / 2010- \\
31 / 08 / 2010\end{array}$ & 4400 & 29.7791 & 1.225 & 0.061 \\
\hline
\end{tabular}

Table 1: Description of selected monthly SCADA datasets from A and B. 
Due to sensor failure and data collection faults SCADA data is not without errors. Such errors will affect power curves and should as far as possible be systematically removed at the outset. Following [30], data are preprocessed according to criteria like timestamp mismatches, out of range values, negative power values, and turbine power curtailment to remove misleading data. Table 1 summarises the datasets of turbine A and B. Set A starts with time stamp "1/02/2010 00:00 AM' and ends at time stamp " 28/02/2010 23:50 PM' and contains 4032 measured values which reduced to 1205 data points after pre-processing, while B starts with time stamp " $1 / 08 / 2010$ 00:00 AM" and ends at time stamp "31/08/2010 23:50 PM' and contains 4400 which became 2068 data points after pre-processing. These pre-processed data points are then used for further analysis. Figures 1 and 3 show the monthly unfiltered power curve data for the turbines at sites A and B respectively. Figures 2 and 4 show filtered monthly power curves for the respective turbines. Note that this data has not in any way been adjusted to reflect air temperature. As already mentioned, the mean absolute value of the air density for each of the WT has been calculated (Table 1). The differences between the months can be useful in understanding the analysis of power curve fitting dealt with later in the paper.

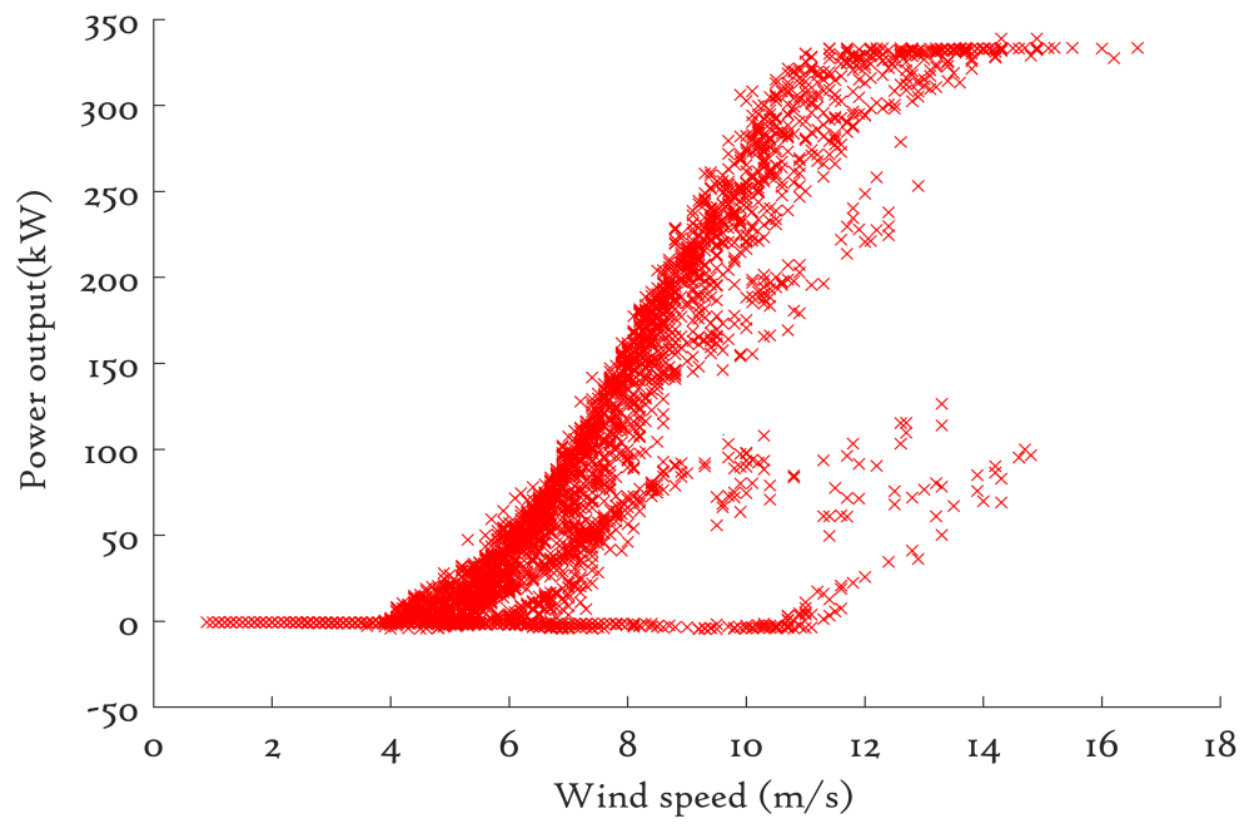

Fig 1: Raw data for turbine A dataset 


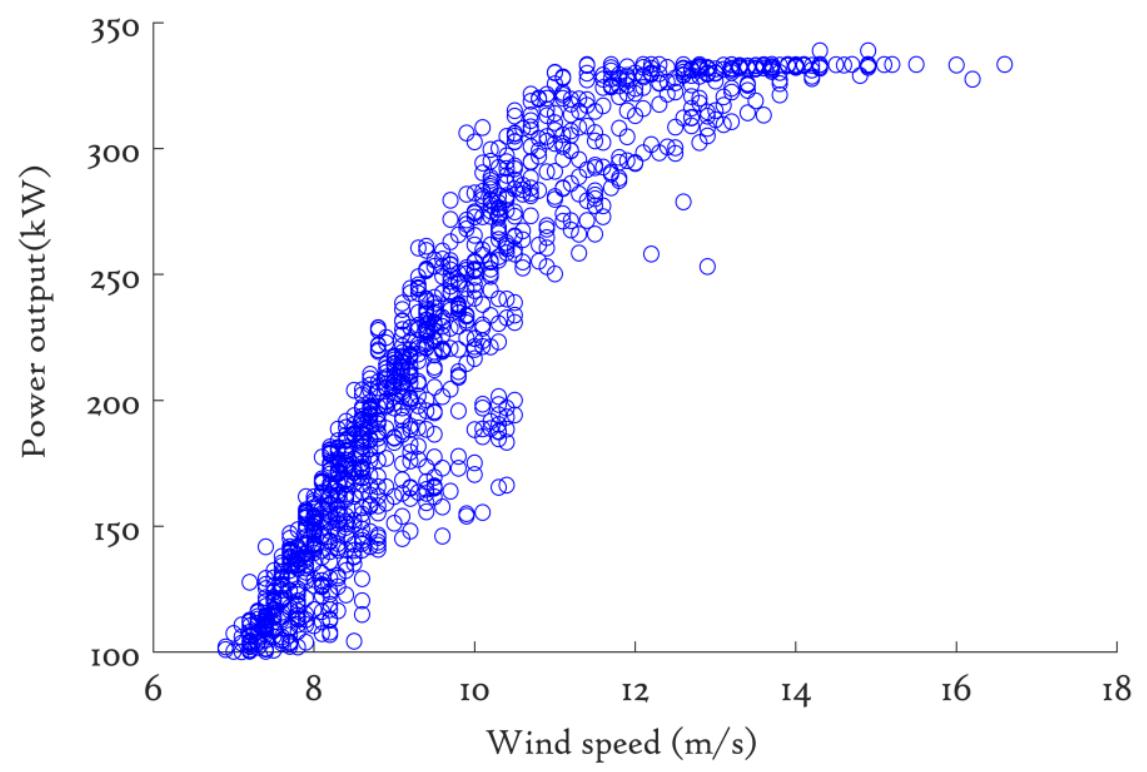

Fig 2: Filtered \& corrected data for turbine A

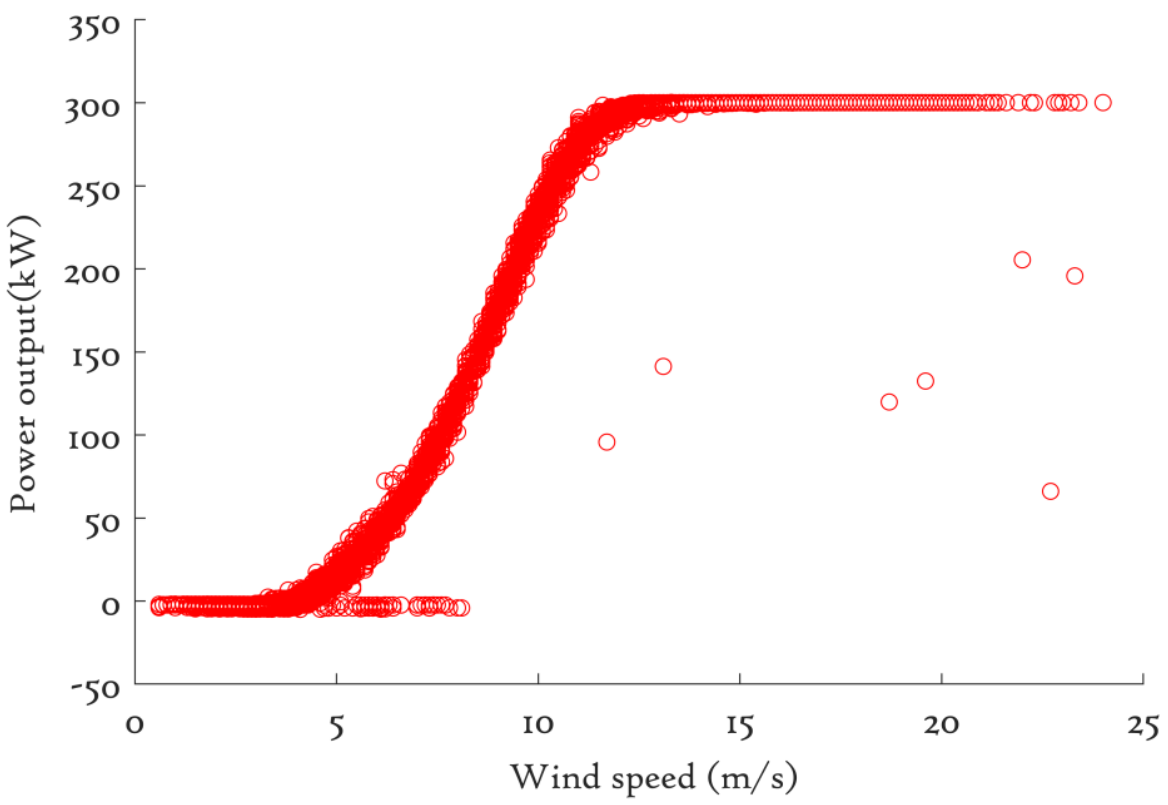

Fig 3: Raw data for turbine B dataset 


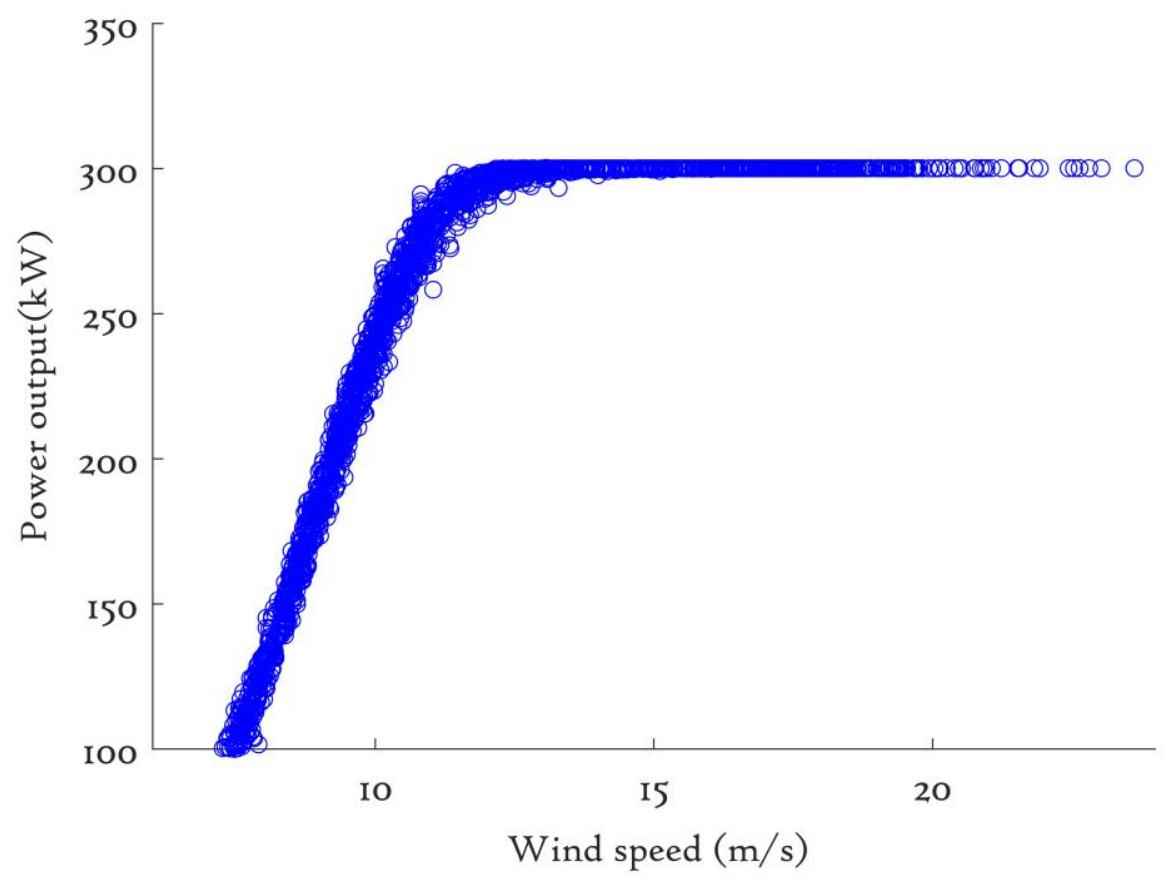

Fig 4: Filtered \& corrected data for turbine B

\section{Gaussian Process power curve modeling and inclusion of air density}

A Gaussian Process (GP) is a nonparametric machine learning algorithm based on a collection of random variables with a joint Gaussian distribution. Using a finite number of measured data points, the role of multivariate Gaussian distribution is to govern and or control the manipulation of the GP models. A GP is mathematically defined by its mean function and covariance functions (or kernel) as given in equation (3),

$$
Y \sim G P(\mu, \Sigma)
$$

where $\mu$ is the mean function and $\sum$ is the covariance function; it has an associated probability density function:

$$
P(x ; \mu, \Sigma)=\frac{1}{(2 \pi)^{\frac{n}{2}}|\Sigma|^{\frac{1}{2}}} \exp \left\{-\frac{1}{2}(x-\mu)^{T} \Sigma^{-1}(x-\mu)\right\}
$$

where $\left|\sum\right|$ is defined as a determinant of $\sum, n$ is the dimension of random input vector $x$, and $\mu$ is

mean vector of $x$. The term under the exponential, i.e. $\frac{1}{2}(x-\mu)^{T} \Sigma^{-1}(x-\mu)$, is an example of a quadratic form. A 1-dimensional Gaussian Process model is a special case where $x$ is a scalar. The general covariance matrix, $K$, gives the variance of each variable along the leading diagonal, 
and the off-diagonal elements measure the correlations between the different variables mathematically described as follows,

$$
K=\left[\begin{array}{ccc}
k_{11} & \cdots & k_{1 n} \\
\vdots & \ddots & \vdots \\
k_{n 1} & \cdots & k_{n}
\end{array}\right] \quad \text { where } k_{i j}=k\left(x_{i}, x_{j}\right)
$$

$K$ is of size $n \times n$, where $n$ is the number of input parameters considered, and it must be symmetric and positive semidefinite i.e. $\sum_{i j}=\sum_{j i}$. For this study, $n=2$ with $x$ accommodating wind speed and air density values incorporated in order to facilitate analysis the effect of air density on predicted power curves.

The covariance function characterizes correlations between different points, i.e., measures the similarity between these points, and is chosen such that it reflects the prior beliefs about the function to be learned and hence considered to be crucial to the performance of GP models. The choice of kernel functions explains both the space of functions that can be generated by the GP and a probability measure on that space. There are various available covariance functions described in [31], and selection is based on given problems and the nature of the datasets. The squared exponential covariance function $\left(k_{S E}\right)$ is commonly applied which for any finite collection of inputs $\{x 1, x 2, \ldots ., x n\}$ is defined as:

$$
k_{S E}\left(x, x^{\prime}\right)=\sigma_{f}^{2} \exp \left(-\frac{\left(x-x^{\prime}\right)^{2}}{2 l^{2}}\right)
$$

This squared exponential covariance function will be used in this paper. SCADA data from the wind turbine comes with measurement errors, so it is desirable to add a noise term to the covariance function to improve the accuracy of the GP model. Hence equation (5) is modified to be:

$$
k_{S E}\left(x, x^{\prime}\right)=\sigma_{f}^{2} \exp \left(-\frac{\left(x-x^{\prime}\right)^{2}}{2 l^{2}}\right)+\sigma_{n}^{2} \delta\left(x, x^{\prime}\right)
$$

where $\sigma_{f}^{2}$ and $l$ are known as the hyper-parameters. $\sigma_{f}^{2}$ signifies the signal variance and $l$ is a characteristic length scale which describes how quickly the covariance decreases with the distance between points. $\sigma_{n}$ is the standard deviation of the noise fluctuation and gives information about model uncertainty. $\delta$ is the Kronecker delta.

In GP models, the mean function is set to zero by appropriate renormalizing of the variable x. GP models are characterized by the hyperparameters that determine the covariance function; these 
have a substantial impact on the posterior model accuracy. A GP model estimates these parameters by maximizing the marginal log-likelihood of the model which depends on the nature of data, see [31]. The proper optimization of these hyperparameters ensures GP power curve model accuracy and is described as follows. For a given training dataset, $\mathrm{A}=\left\{\left(x_{i}, y_{i}\right), i=1, \ldots \ldots, N\right\}$ of $n$ observations, calculated values of mean and variance are required in order to model the GP power curve. Here $x$ is the input vector of dimension $\mathrm{D}$, and $y$ is the scalar output. The given set of input datasets is denoted by a $A \times n$ matrix. With target output collected in a vector y, we can define the relationship as $A=(X, y)$. A GP regression model is used to solve the relationship between input and targets value which is modelled as:

$$
y_{i}=f\left(x_{i}\right)+\epsilon_{i}
$$

The above equation is theoretically used to define the underlying function of the data modeled where $x$ are values from the training datasets and $\epsilon$ is Gaussian white noise of variance $\sigma_{n}^{2}$ such that, $\epsilon=N\left(0, \sigma_{n}^{2}\right)$. And the prior to $y$ becomes:

$$
\begin{gathered}
E|\mathrm{y}|=\mathrm{E}|f+\epsilon|=0 \\
\operatorname{cov}|\mathrm{y}|=K|X, X|+\sigma_{n}^{2} I
\end{gathered}
$$

The prior distribution is useful in providing essential information about the uncertain parameters. This prior distribution with the probability distribution of future data is used to develop the posterior distribution which is helpful for inference and in any decisions involving uncertain parameters. To estimate the output $f$, for a new input $x^{*}$, the distribution can be written as :

$$
\left(\begin{array}{c}
y \\
f^{*}
\end{array}\right) \sim N\left(0,\left[\begin{array}{cc}
K(X, X)+\sigma_{n}^{2} I & k\left(X, x^{*}\right) \\
k\left(x^{*}, X\right) & k\left(x^{*}, x^{*}\right)
\end{array}\right]\right)
$$

where $k\left(X, x^{*}\right)=k\left(x^{*}, X\right)^{T}=\left[k\left(x_{1}, x^{*}\right), k\left(x_{2}, x^{*}\right), \ldots . k\left(x_{n}, x^{*}\right)\right]$, which is for simplicity denoted by $k^{*}$. Then, from the joint Gaussian distribution, the estimation of target values is given by:

$$
\begin{gathered}
\overline{f *}=k_{*}^{T}\left(K+\sigma_{n}^{2} I\right)^{-1} y \\
\operatorname{Var}[f *]=\mathrm{k}\left(x^{*}, x^{*}\right)-k_{*}^{T} k^{*}\left(K+\sigma_{n}^{2} I\right)^{-1}
\end{gathered}
$$


The obtained posterior variance $(\operatorname{Var}[f *])$ is inversely proportional to the distance between test and training data points while estimation of the mean $(\overline{f *})$ is a linear combination of the output $y$ in which linear weights are defined $k_{*}^{T}\left(K+\sigma_{n}^{2} I\right)^{-1}$.

Using the filtered SCADA datasets (of Figures 2 and 4), power curves have been constructed using GP models described above (realized in MATLAB) for the different approaches to air density correction, and the results are illustrated in Figures 5 and 6. As already described in section 3, the data for wind turbine B has limited scatter (see Figure 4) and results in a smooth GP power curve, see Figure 5. In contrast, SCADA data for wind turbine A is more scattered (due to extremely low temperatures) and also has fewer data points at the rising section of the power curve. This issue along with cubic inversion problem (described in next section) affects the GP model fitting accuracy and increases the computation cost, so striking a balance between the number of data points used and computation cost is critical for effective GP modeling. For these reasons, the data sets will be restricted to a wind speed range of 8 to $14 \mathrm{~m} / \mathrm{s}$, as outlined below. For a wind speed range of 8 to $14 \mathrm{~m} / \mathrm{s}$, the two data sets have almost same number of data points. GPs work well with data sets of limited size; this is because the process involves inverting a matrix of dimension equal to the number of data points. SCADA information is gathered at 10-minute intervals over extended timeframes, so this can be an issue. The posterior conditional distribution for a given observation mathematically is defined in [32], and there is a covariance matrix component, as given by equation (13), associated with the inverse matrix operation which leads to the mathematical challenge of inverting an $n \times n$ matrix (and this goes approximately with $O\left(\mathrm{n}^{3}\right)$, where $n$ is the number of data points). 

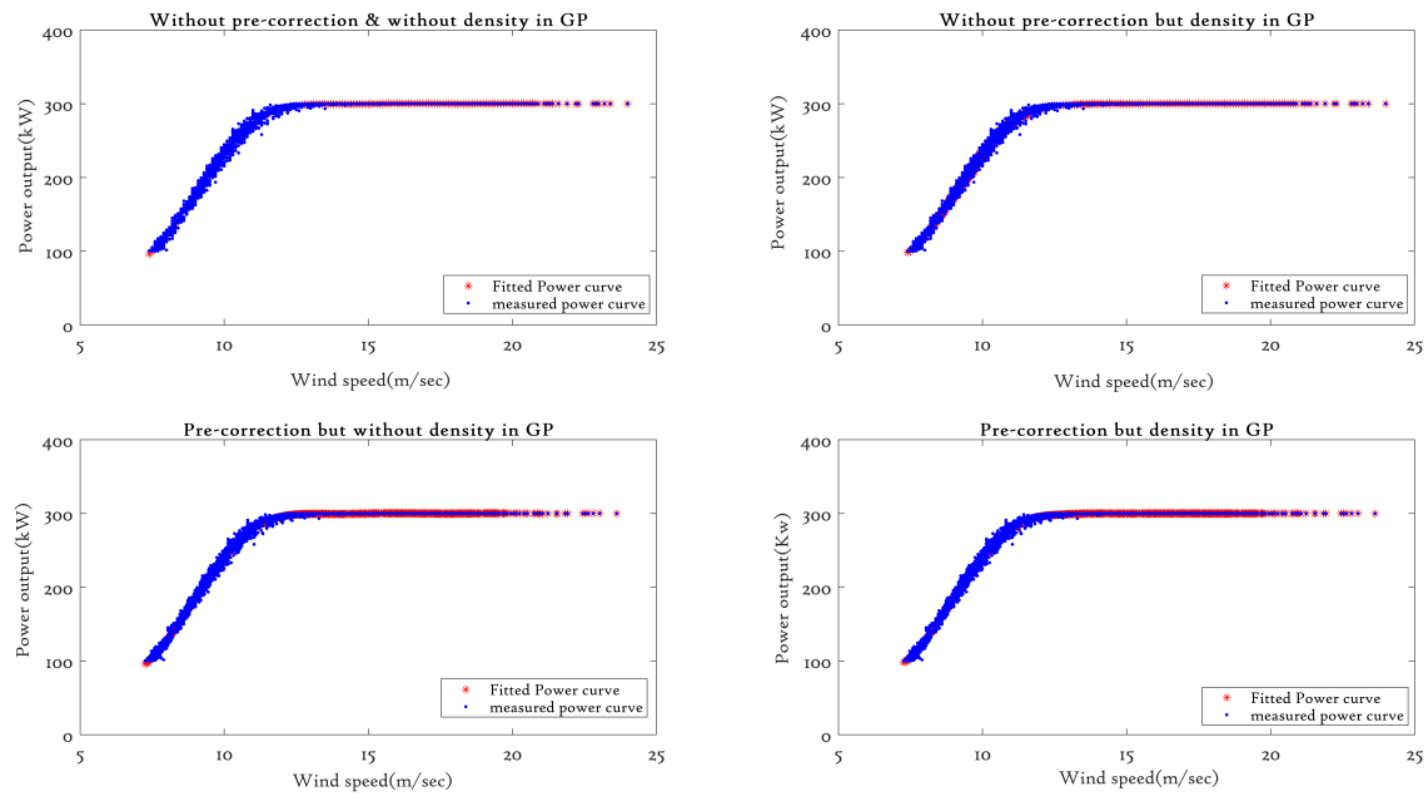

Fig 5: GP power curve fitting for different air density approaches using site B data
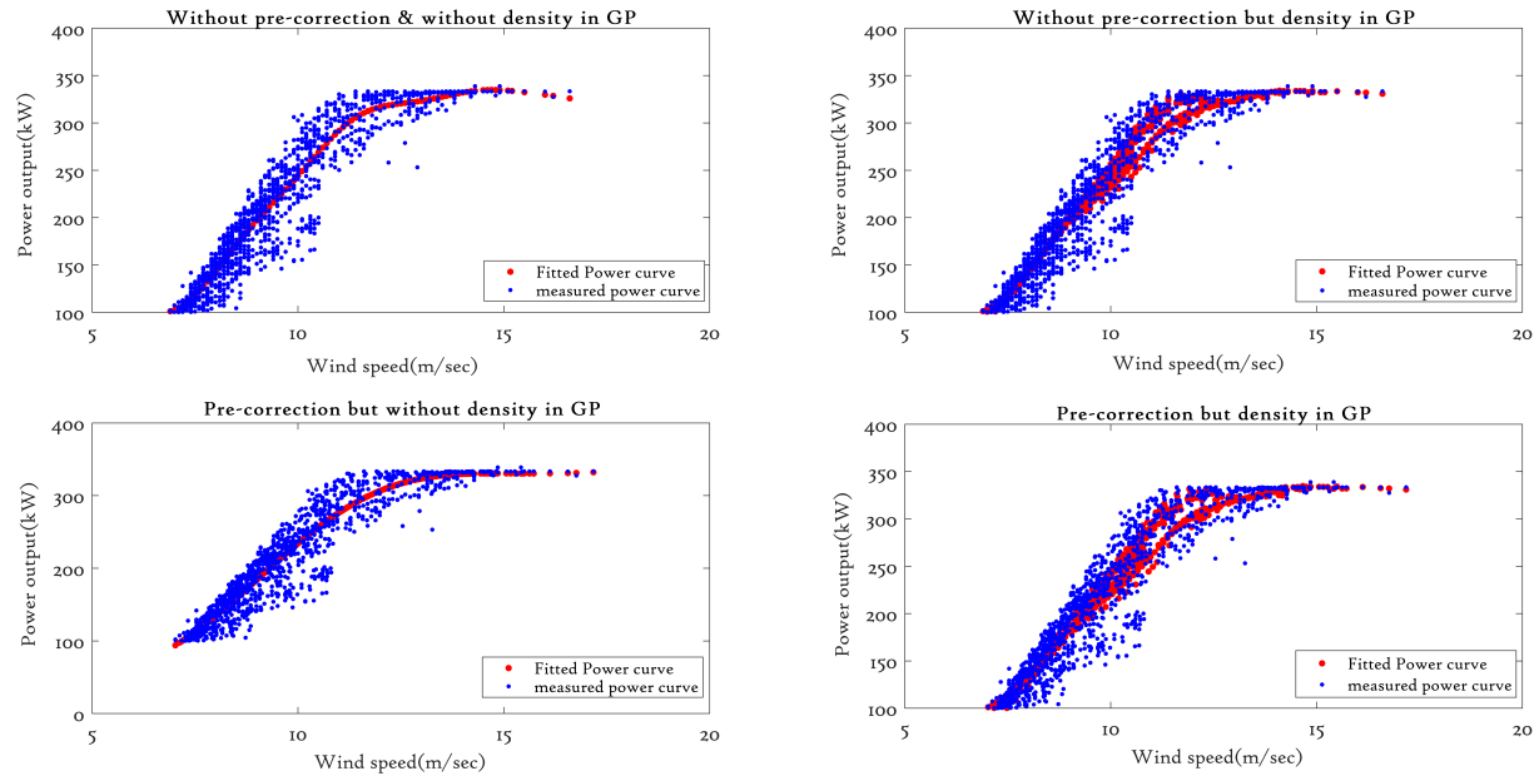

Fig 6: GP power curve fitting for different air density approaches using site A data

If $n$ is too large, then the computation of the $n \times n$ matrix becomes problematic and leads to GP model inaccuracy.

$$
Y_{x^{*}} \mid Y_{x}=y_{x} \sim G P\left[\begin{array}{c}
\mu\left(x^{*}\right) \sum\left(x^{*}, x\right) \sum(x, x)^{-1}\left(y_{x}-\mu(\mathrm{x})\right), \\
\sum\left(x^{*}, x^{*}\right)-\sum\left(x^{*}, x\right) \sum(x, x)^{-1} \sum\left(\mathrm{x}, x^{*}\right)
\end{array}\right]
$$


State-space representations of GPs are being introduced to address this cubic problem, [33,34]. Moreover, in [35], two parallel GP regression methods that exploit low-rank covariance matrix approximations for distributing the computational load among parallel machines are being used to solve these problems, but these still require high processing power and computational cost in dealing with large datasets. For effective analysis, GP model accuracy has been assessed (section 5) for a limited wind speed range of 8 to $14 \mathrm{~m} / \mathrm{s}$; this results in the number of data points used in the two cases being almost equal. Moreover, for this range of wind speeds data from turbine $\mathrm{A}$ is less scattered. The accuracy of a GP power curve depends upon the quantity and quality of the data. A low number of power-wind speed pairs may not give a smooth power curve while a high number is not desirable because of the numerical challenge of calculating the GP, [36].

\section{Gaussian Process model accuracy assessment for different air density approaches}

In this section, the accuracy of the GP power curves with the different approaches to air density compensation are assessed using the residual distributions and a range of statistical measures (MAE, MSE, MAPE, RMSE, $R^{2}$ ). Confidence intervals are utilized to portray how individual air density approaches affects the GP model uncertainty. As mentioned above, to limit the size of the data set, the analysis will be restricted to the wind speed range of 8 to $14 \mathrm{~m} / \mathrm{s}$ since the number of data points are sufficient within this range and also the number of data points resulting for sites $\mathrm{A}$ and $\mathrm{B}$ are almost the same and less scattered. This has the further advantage that the measurement errors (assumed to be constant in the GP) are reasonably consistent. The mean absolute value of the air density correction, has been recalculated for this restricted wind speed range, and is shown in Table 2.

\begin{tabular}{cccc}
\hline Site & $\begin{array}{c}\text { Standard } \\
\text { density }\end{array}$ & $\begin{array}{c}\text { Mean absolute density } \\
\text { difference }\end{array}$ & $\begin{array}{c}\text { Total number of data } \\
\text { points used }\end{array}$ \\
\hline A & 1.225 & -0.099 & 1114 \\
\hline B & 1.225 & 0.062 & 1116 \\
\hline
\end{tabular}

Table 2: The Mean absolute value of standard air density correction for restricted range data sets 


\subsection{Uncertainty Assessment using confidence intervals}

A GP power curve model intrinsically represents fitting errors and thus model accuracy. An accurate assessment of a GP power curve can be done using confidence intervals (CIs). These GP confidence intervals provide information on the uncertainty surrounding an estimation but are itself a model-based estimate, [31]. Confidence intervals are a useful measure of uncertainty and the precision of model estimates. Data points that lie outside of the confidence intervals can be considered anomalous, signifying a potential malfunction of the wind turbine. For example, authors of [37] used the intrinsic confidence interval of a GP model to detect anomalous operation caused by yaw misalignment in which $95 \%$ CIs were used to determine anomalous data point values. In addition, a Fisher test and associated 3p-values were used with a threshold of 0.008 applied to filter the individual p-values. In this way, the GP CIs were able to detect the anomalous data caused by yaw error. The standard deviation is the square root of the variance of the predicted function $(\operatorname{Var}[f *])$ (of equation 12) and used to estimate the confidence intervals of the GP power curve model using equation (14),

$$
C I s=\overline{f * \pm} 2 \sqrt{\operatorname{Var}}[f *]
$$

Equation (15) suggest that CIs represent the pointwise mean plus and minus two times the standard deviation for given input value (corresponding to the $95 \%$ confidence region which represents the significance level of 0.05), for the prior and posterior respectively. Confidence intervals have been calculated for the restricted data sets for sites A and B as outlined above. Based on these it is found that the traditional approach to air density correction does not give the most accurate GP model. The most accurate approach is not to undertake air density pre-correction but to include air density within the GP model, and this further verified by the results shown in Figures 7 and 8, where the confidence interval plotted as a function of the wind speed. 


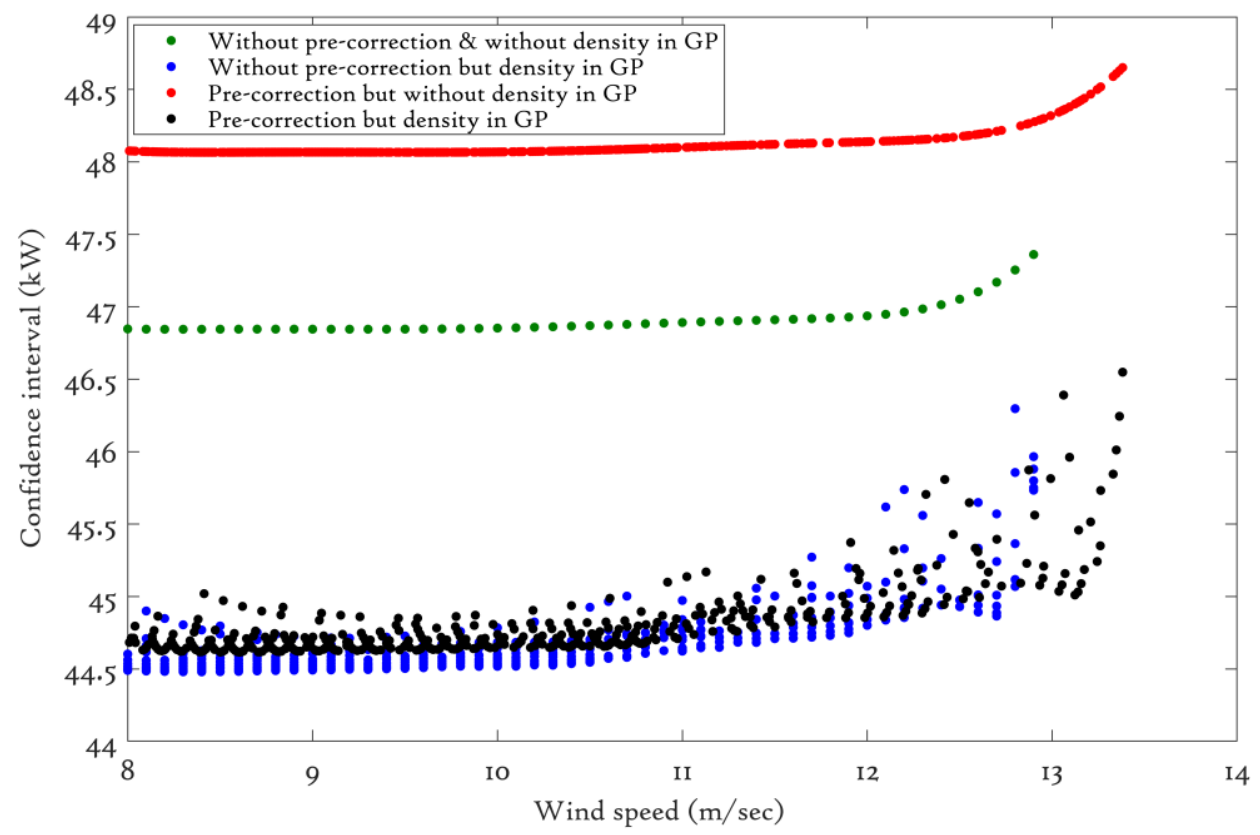

Fig 7: Uncertainty assessment in terms of confidence intervals for different air density approaches with limited data set for site A

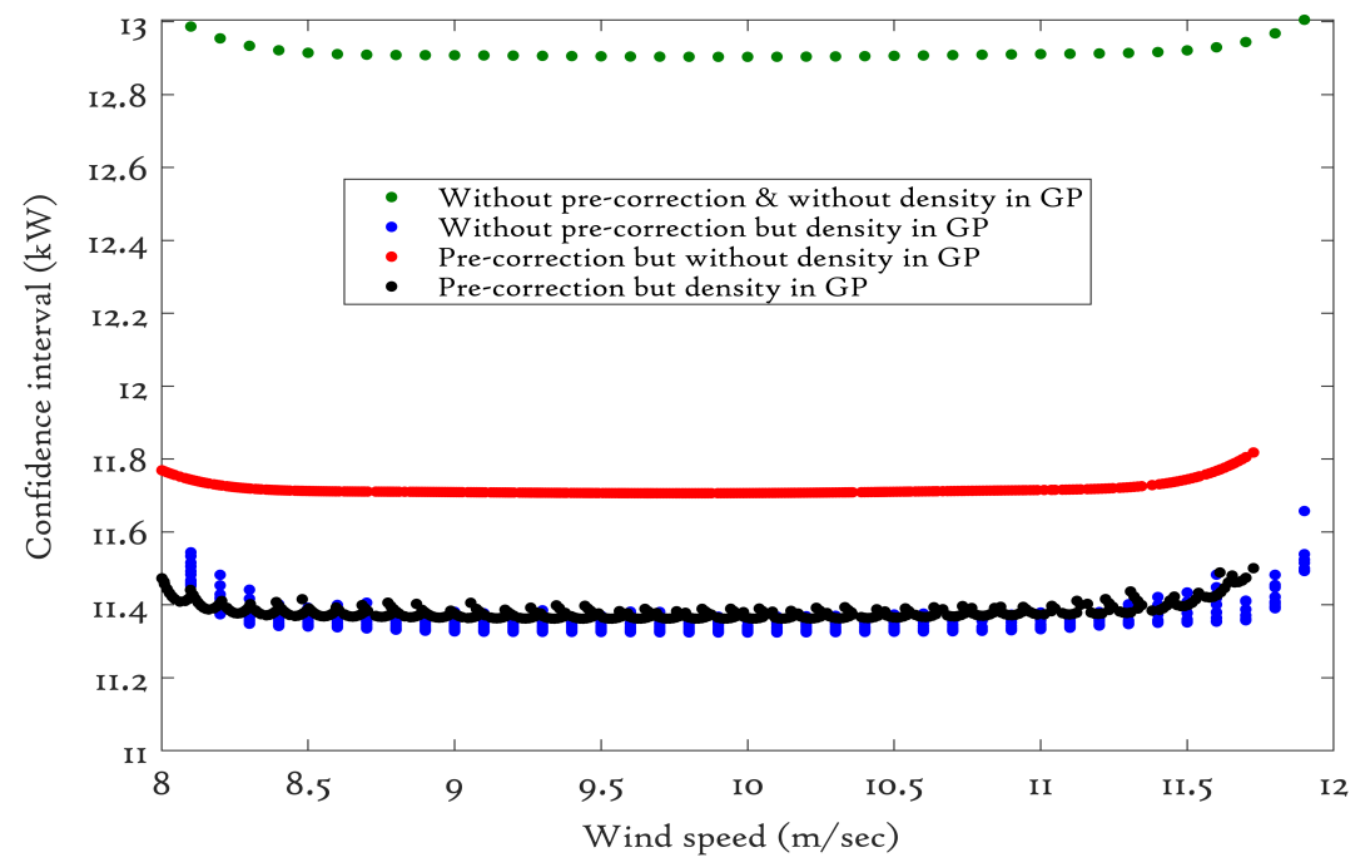

Fig 8: Uncertainty assessment in terms of confidence intervals for different air density approaches with limited data set for site B 


\subsection{Performance Error Metrics}

Five goodness-of-fit indicators, namely mean absolute error (MAE), mean square error (MSE), mean absolute percentage error (MAPE), root mean square error (RMSE) and coefficient of determination $\left(R^{2}\right)$ are calculated. They are described as follows.

The difference between the measured and estimated values expressed by mean absolute error (MAE):

$$
\operatorname{MAE}=\frac{\sum_{\mathrm{i}=1}^{\mathrm{n}} \operatorname{abs}\left(\mathrm{y}_{\mathrm{i}}{ }^{\prime}-\mathrm{y}_{\mathrm{i}}\right)}{\mathrm{n}}
$$

In terms of residuals, $\quad \mathrm{MAE}=\frac{\sum_{\mathrm{i}=1}^{\mathrm{n}}(e)}{\mathrm{n}}$

where residuals are defined as $e=y-y^{\prime}$

To quantify the magnitude of the residuals (i.e., the difference between observed and modeled values), the root mean square error (RMSE) is commonly used, defined as:

$$
\operatorname{RMSE}=\sqrt{\frac{\sum_{\mathrm{i}=1}^{\mathrm{n}}\left(\mathrm{y}_{\mathrm{i}}^{\prime}-\mathrm{y}_{\mathrm{i}}\right)^{2}}{\mathrm{n}}}
$$

where $y^{\prime}$ are the GP predicted values for $n$ different predictions, and $y$ are the measured values.

In terms of residuals, this is: $\quad$ RMSE $=\sqrt{\frac{\sum_{\mathrm{i}=1}^{\mathrm{n}}(\mathrm{e})^{2}}{\mathrm{n}}}$

The coefficient of determination $\left(R^{2}\right)$ quantifies how close the data are to the fitted regression, and calculated as the square of the correlation between predicted output and measured values (hence always in the range from 0 to 1 with values closer to 1 indicates better fitting of the model to the

data). It is defined as $R^{2}=1-\frac{S S E}{T S S}$, where SSE is the sum of squared errors and TSS is the total sum of squares. Another statistical measure, the mean absolute percentage error (MAPE) is an alternative a measure of prediction accuracy of a model, [38].

In short, the five statistical measures: mean absolute error (MAE); mean square error (MSE); mean absolute percentage error (MAPE); root mean squared error (RMSE), and coefficient of determination $\left(R^{2}\right)$ are considered as useful indicators of the goodness of model fitting. They are used here to assess the accuracy of the GP power curve models for the different air density 
correction approaches. The results of these statistical measures suggests that without precorrection but adding density into GP model approach gives the most accurate result as compared to other GP models and results as shown in table 3. The conclusion is that with a GP model, precorrection does more harm than good, and this is assumed to be due to the imperfect nature of the pre-correction.

\begin{tabular}{|c|c|c|c|c|c|c|c|c|c|c|}
\hline \multirow[t]{2}{*}{ GP models } & \multicolumn{5}{|c|}{ WT- A } & \multicolumn{5}{|c|}{ WT-B } \\
\hline & MAE & MSE & MAPE & RMSE & $R^{2}$ & MAE & MSE & MAPE & RMSE & $R^{2}$ \\
\hline $\begin{array}{l}\text { No pre- } \\
\text { correction and } \\
\text { air density not } \\
\text { included in the } \\
\text { GP model }\end{array}$ & 18.012 & 568.075 & 9.439 & 23.834 & 0.878 & 5.196 & 43.094 & 2.469 & 6.564 & 0.982 \\
\hline $\begin{array}{l}\text { No pre- } \\
\text { correction but } \\
\text { with air density } \\
\text { included within } \\
\text { the GP model }\end{array}$ & 16.813 & 506.967 & 8.958 & 22.515 & 0.891 & 4.626 & 33.012 & 2.204 & 5.745 & 0.986 \\
\hline $\begin{array}{l}\text { Pre-correction } \\
\text { applied but } \\
\text { without air } \\
\text { density in the } \\
\text { GP model }\end{array}$ & 18.501 & 598.094 & 9.680 & 24.456 & 0.872 & 4.736 & 35.463 & 2.251 & .955 & 0.985 \\
\hline $\begin{array}{l}\text { With pre- } \\
\text { correction and } \\
\text { air density } \\
\text { included with } \\
\text { the GP }\end{array}$ & 16.868 & 510.016 & 8.990 & 22.583 & 0.891 & 4.648 & 33.344 & 2.215 & 5.774 & 0.986 \\
\hline
\end{tabular}

Table 3: Statistical measures of GP fitted models under different air density approaches

\subsection{Residual Distribution analysis using QQ Plot}

The distribution of the residuals can also be informative in the context of GP models. Figures 9 and 11 are the time series of residuals. The effect of air density on the GP model residual distribution is significant and seen by distribution fits for all the data sets considered as shown in Figures 10 and 12. A quantile-quantile or QQ plot is a simple graphical method used to compare collections of data with possible theoretical distributions and can help in distribution function identification [39]. QQ plots are a useful tool used to compare the shapes of distributions, providing a graphical view of how properties such as shape, location, size, and skewness are similar or different. 

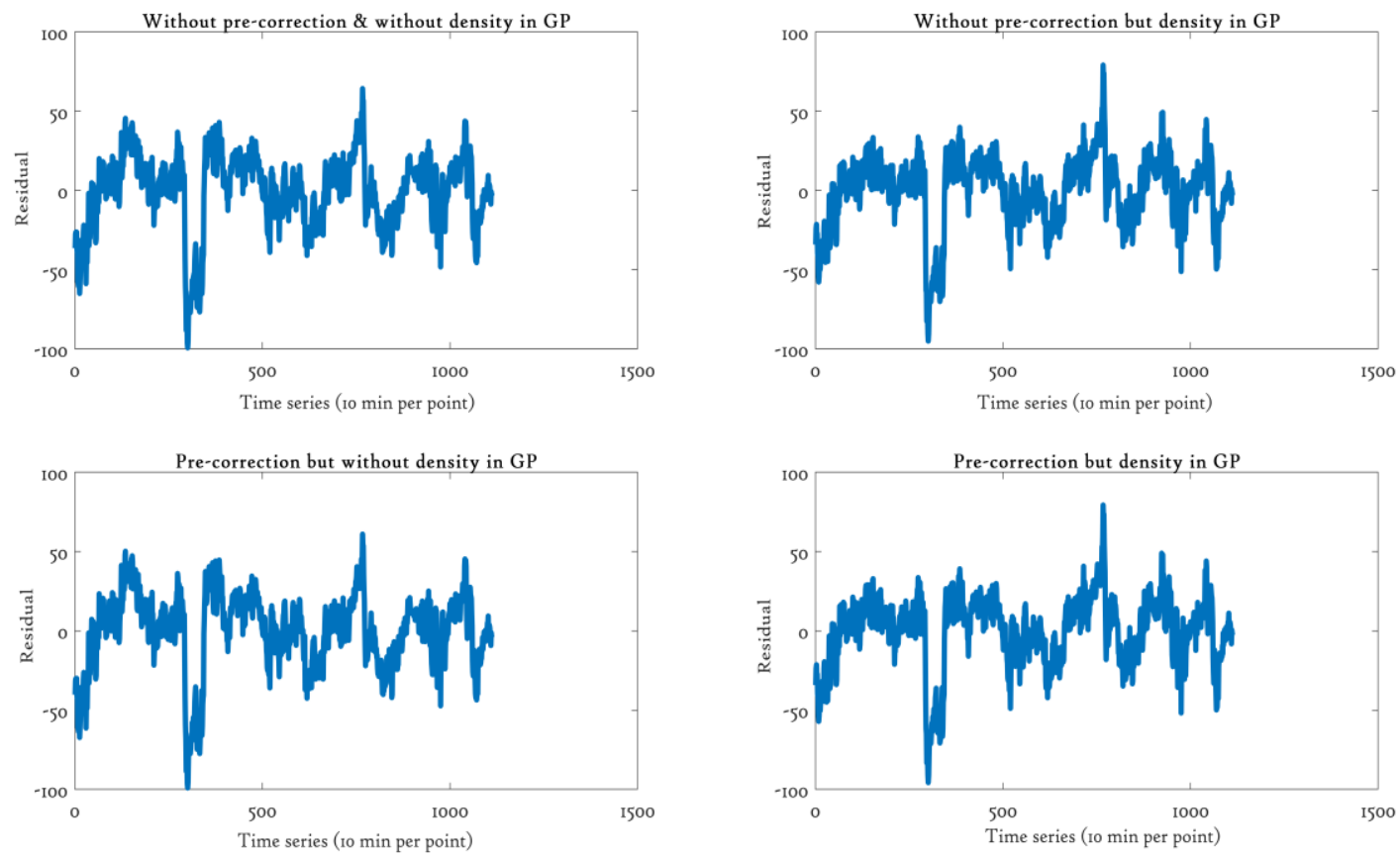

Fig 9: Residual for restricted datasets, A
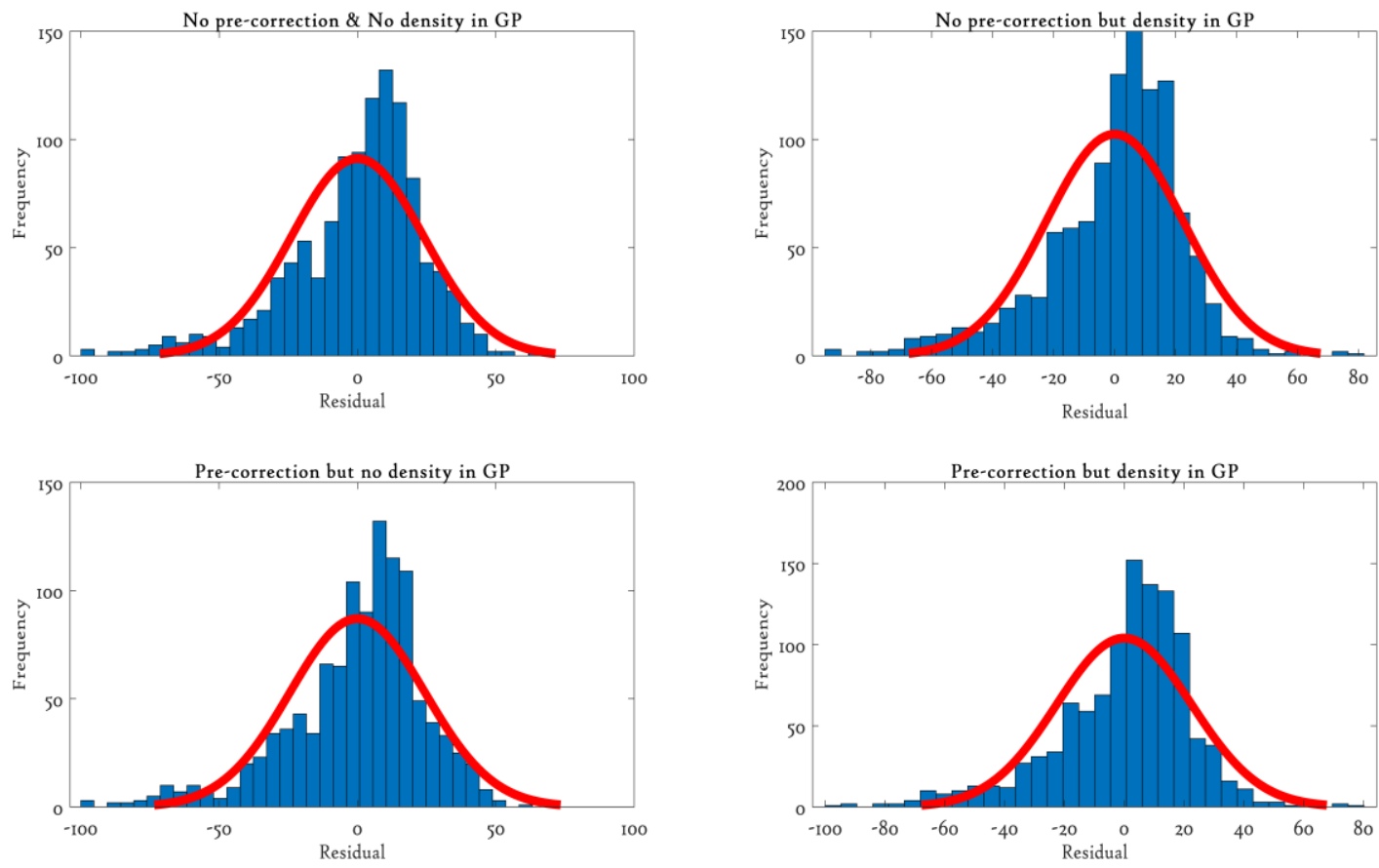

Fig 10: Residual histogram with distribution fit, A 

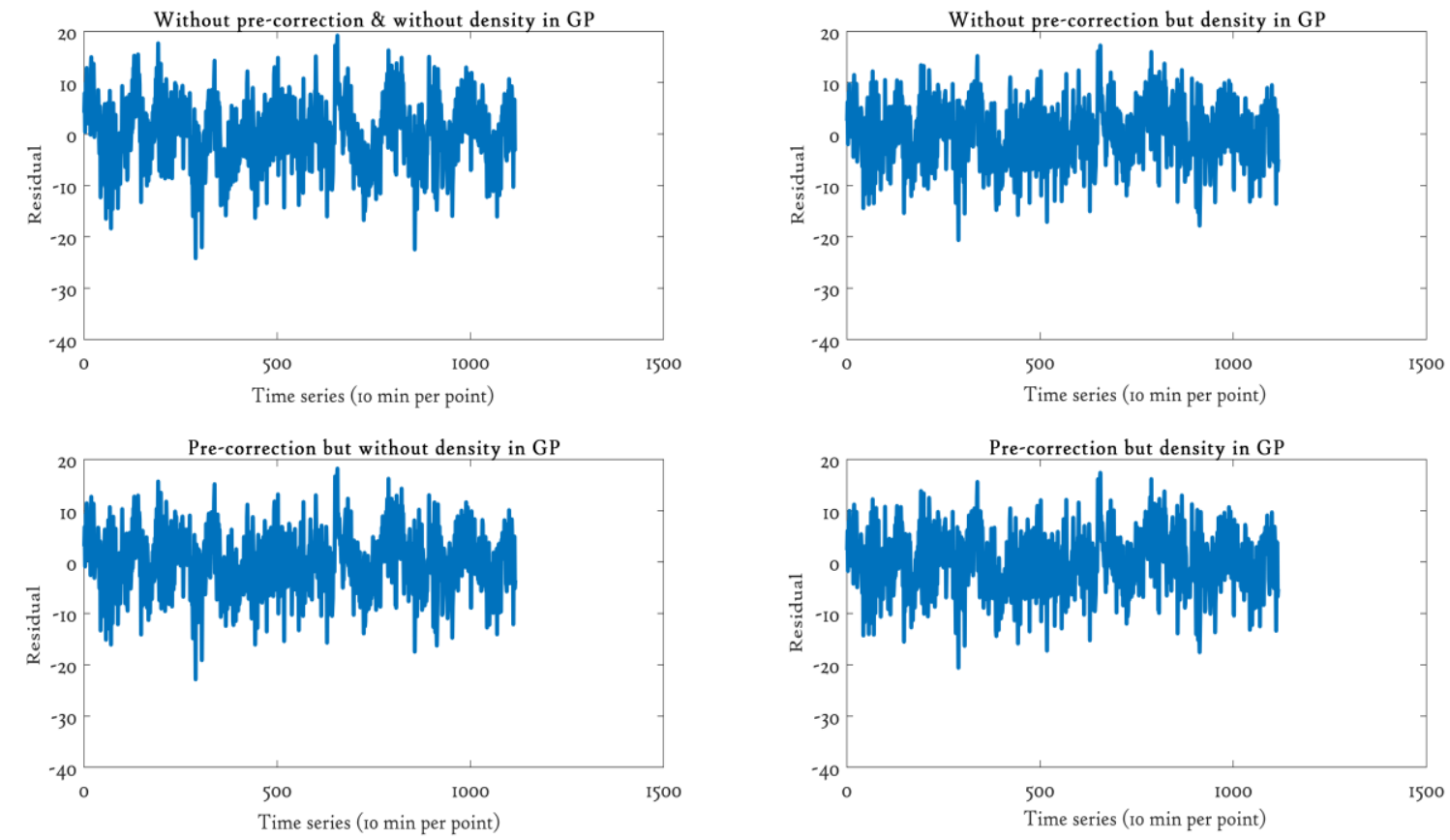

Fig 11: Residual for restricted datasets, B
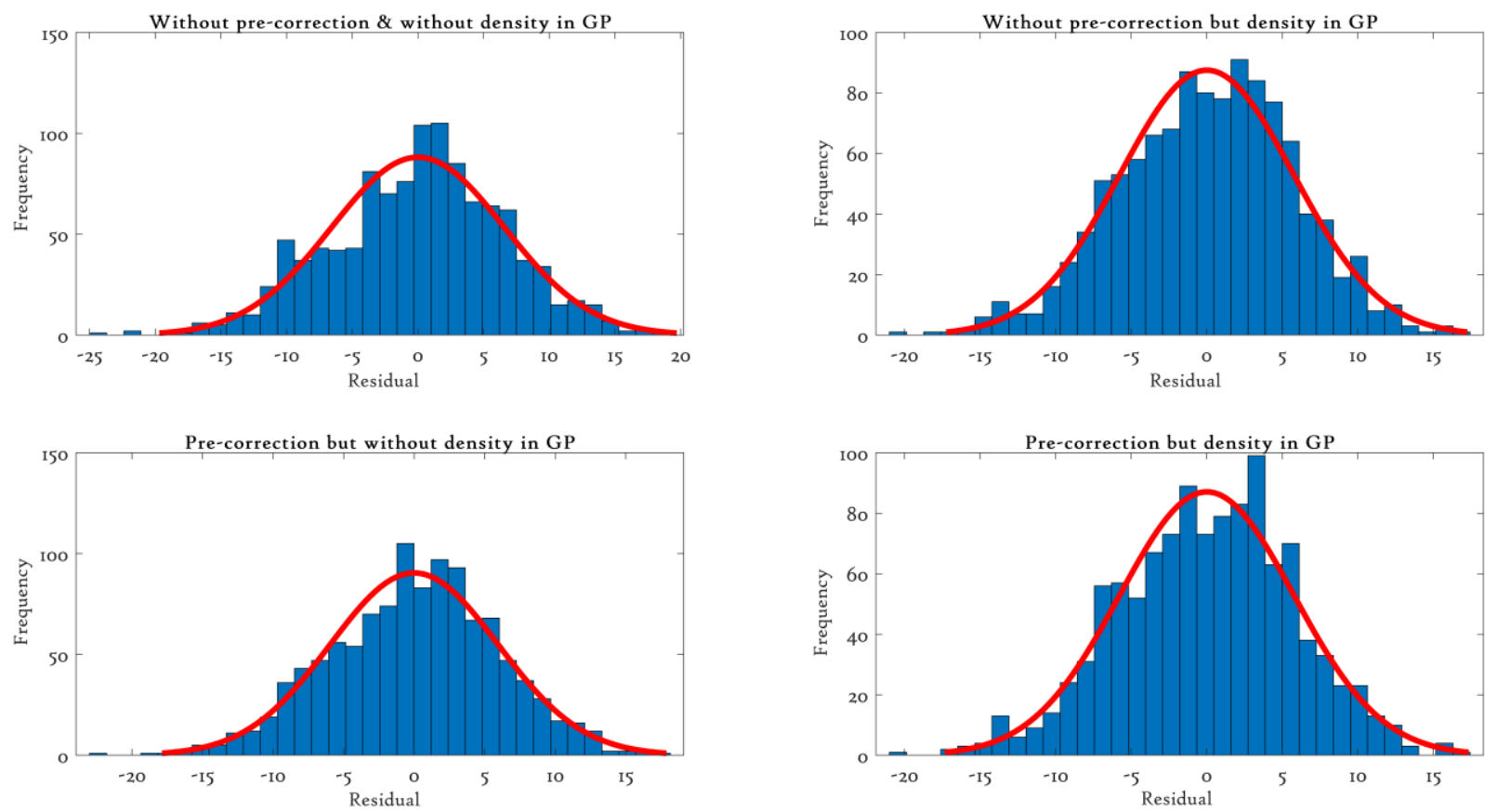

Fig 12: Residual histogram with distribution fit, B

QQ plots are commonly used to compare two samples of data and their underlying distributions and thus used in this study for GP power curve distribution analysis for different air density compensation techniques [40]. For GP models, the residuals should be Gaussian distributed. An 
ideal QQ plot would be a straight line with unity gradient. QQ plots comparing the residual distribution with a Gaussian distribution have been calculated for the four different cases and assessed regarding RMSE differences from the ideal unity gradient line. Table 4 shows that the case of no pre-correction but with air density include within the GP model results in residuals most closely conforming to a Gaussian distribution for both wind turbines A and B. This adds to the case that the GP model is more accurate with no pre-correction but with air density included within the GP power curve model.

\begin{tabular}{cccc}
\hline Sr.no & GP models & site A & site B \\
\hline $\mathbf{1}$ & $\begin{array}{c}\text { No pre-correction and air density not } \\
\text { included in the GP model }\end{array}$ & 2.292 & 1.530 \\
\hline $\mathbf{2}$ & $\begin{array}{l}\text { No pre-correction but with air density } \\
\text { included within the GP model }\end{array}$ & $\mathbf{0 . 6 4 1}$ & \\
\hline $\mathbf{3}$ & Pre-correction applied but without air & & \\
\hline & density in the GP model & 2.020 & 0.476 \\
\hline & With pre-correction and air density included & 0.650 & \\
\hline
\end{tabular}

Table 4: RMSE for different density approaches from QQ plot.

\section{Conclusion and Discussion}

Air density corrections are essential for accurate power curve modeling. With the possibility to include air desity within GP models, various alternative approaches are possible. This paper has proposed four air density correction approaches and assessed their differeing impact on GP WT power curve model accuracy. Two SCADA datasets were used that satisfied the IEC standard guideline which states that the air density correction should only be applied when the site density differs from the standard value $\left(1.225 \mathrm{~kg} / \mathrm{m}^{3}\right)$ by more than $0.05 \mathrm{~kg} / \mathrm{m}^{3}$. The results demonstrate that the best option is not to use the standard IEC correction before applying the GP model. Error performance metrics suggest that separately adding density directly into the GP power curve model, without any pre-correction gives most accurate results, see table 3. Furthermore, QQ plot analysis, as provided in table 4, confirms this result. In short, from this paper we conclude that air density corrections can be significant; the best option is not to apply the standard IEC correction before applying the GP model. This will not only help in power curve 
modeling but also supports the construction of robust algorithms for anomaly detection via confidence intervals. It should be noted that SCADA data used in this study assumes the turbine to have single rated power value. However, if the SCADA data contains multiple rated power values, then separate GP power curves can be constructed for the different rated power values. Future work will involve using this outcome for developing improved GP model based anomaly detection for wind turbine condition monitoring.

\section{Acknowledgments}

This project has received funding from the European Union's Horizon 2020 research and innovation programme under the Marie Sklodowska-Curie grant agreement No 642108.

Conflict of interest: The authors have declared no conflicts of interest for this article.

\section{References}

1. Morshedizadeh M, Kordestani M, Carriveau R, Ting D, Saif M. Improved power curve monitoring of wind turbines. Wind Engineering.2017; 41(4): 260-271. doi: https://doi.org/10.1177/0309524X17709730.

2. Pelletier F, Masson $\mathrm{C}$, Tahan A. Wind turbine power curve modelling using artificial neural network. Renewable Energy. 2016; 89: 207-214. doi: 10.1016/j.renene.2015.11.065.

3. Jia X, Jin C, Buzza M, Wang W, Lee J. Wind turbine performance degradation assessment based on a novel similarity metric for machine performance curves. Renewable Energy. 2016; 99: 1191-1201, doi: 10.1016/j.renene.2016.08.018.

4. Wang S, Huang Y, Li L, Liu C. Wind turbines abnormality detection through analysis of wind farm power curves. Measurement. 2016; 93: 178-188. doi: 10.1016/j.measurement.2016.07.006.

5. Kusiak A, Verma A. Monitoring Wind Farms with Performance Curves. IEEE Transactions on Sustainable Energy. 2013; 4(1):192-199. doi: 10.1109/TSTE.2012.2212470.

6. Taslimi E, Modiri M, Elias M, Rahim N. Development of an enhanced parametric model for wind turbine power Curve. Applied Energy. 2016;177: 544-552.

doi: $\underline{10.1016 / j . a p e n e r g y .2016 .05 .124 . ~}$ 
7. Shokrzadeh S, Jozani M, Bibeau E. Wind Turbine Power Curve Modeling Using Advanced Parametric and Nonparametric Methods. IEEE Tractions on sustainable energy.2014; 5(4): 1262-1269. doi: 10.1109/TSTE.2014.2345059.

8. Sohoni V, Gupta S, Nema R. A Critical Review on Wind Turbine Power Curve Modelling Techniques and Their Applications in Wind Based Energy Systems. Journal of Energy. 2016; Article ID 8519785. 18 pages. doi: https://doi.org/10.1155/2016/8519785.

9. Üstüntaş T, Şahin A. Wind turbine power curve estimation based on cluster center fuzzy logic modelling. Journal of Wind Engineering and Industrial Aerodynamics.2008;96 (5):611-620. doi: $10.1016 /$ j.jweia.2008.02.001.

10. Yampikulsakul N, Byon E, Huang S, Sheng S, You M. Condition Monitoring of Wind Power System with Nonparametric Regression Analysis. IEEE Transactions on Energy Conversion.2014; 29(2): 288-299. doi: 10.1109/TEC.2013.2295301.

11. IEC Standard 61400-12-1, 2006.Wind Turbines-Part 12-1: Power Performance Measurements of Electricity Producing Wind Turbines.

12. Wang J, Niu T, Lu H, Guo H, Yang W, Du P. An analysis-forecast system for uncertainty modeling of wind speed: A case study of large-scale wind farms. Applied Energy. 2018; 211: 492-512. doi: 10.1016/j.apenergy.2017.11.071.

13. Jafarian M, Soroudi A, Ehsan M. The effects of environmental parameters on wind turbine power PDF curve. In: Proceedings of 2008 Canadian Conference on Electrical and Computer Engineering, Niagara Falls; 2008; pp. 001193-001198. doi: 10.1109/CCECE.2008.4564727.

14. The BARANI. Report on Air density changes due to weather 2016. https://barani.biz/kb/airdensity-changes-due-to-weather/. 2016. Accessed April 10, 2018.

15. Pourrajabian A, Mirzaei M, Ebrahimi R, Wood D. Effect of air density on the performance of a small wind turbine blade: a case study in Iran Journal of Wind Engineering and Industrial Aerodynamics. 2014; 126:1-10, doi: 10.1016/j.jweia.2014.01.001.

16. Zahariea D, Husaru D. Atmospheric air density analysis with Meteo-40S wind monitoring system. In: Proceedings of $21^{\text {st }}$ Innovative Manufacturing Engineering \& Energy International Conference - IManE\&E; 2017. Article no: 07020,pp:6. doi: $\underline{10.1051 / \mathrm{matec} c o n f / 201711207020 .}$.

17. Ciulla G, Dio V, Cascua D, Brano V, Montana F. Effects of the air density value on a wind generator electricity production capability. In: Proceedings of 2016 IEEE 16th International 
Conference on Environment and Electrical Engineering (EEEIC); 2016; Florence, 7-10 June, pp. 1-5. doi: 10.1109/EEEIC.2016.7555512.

18. Jung S, Kwon S. Weighted error functions in artificial neural networks for improved wind energy potential estimation. Applied Energy. 2013; 111:778-90. doi: 10.1016/j.apenergy.2013.05.060.

19. Hau E. Wind turbines. Fundamentals, technologies, application, economics. 3rd edition. New York: Springer publishers; 2013.

20. Yan C, Archer C. Assessing compressibility effects on the performance of large horizontalaxis wind turbines. Applied Energy. 2018; 212:33-45. doi: 10.1016/j.apenergy.2017.12.020.

21. Liu Y, Liu W. Effect of air density on output power of wind turbine. Applied Mechanics and Materials. 2013;(336-338):1114-1117.doi: 10.4028/www.scientific.net/AMM.336-338.1114.

22. Pandit R, Infield D. SCADA-based wind turbine anomaly detection using Gaussian process models for wind turbine condition monitoring purposes. IET Renewable Power Generation.2018 (E-First). doi: 10.1049/iet-rpg.2018.0156.

23. Yang Y, Li S, Li W, Qu M. Power load probability density forecasting using Gaussian process quantile regression. Applied Energy. 2018; 213:499- 509. doi: https://doi.org/10.1016/j.apenergy.2017.11.035.

24. Chen N, Qian Z, Nabney I, Meng X. Wind Power Forecasts Using Gaussian Processes and Numerical Weather Prediction. IEEE Transactions on Power Systems. 2014; 29(2): 656-665. doi: 10.1109/TPWRS.2013.2282366.

25. Yan J, Li K, Bai Er-Wei, Deng J, Foley A. Hybrid Probabilistic Wind Power Forecasting Using Temporally Local Gaussian Process. IEEE Transactions on Sustainable Energy. 2016;7(1): 87-95. doi:10.1109/TSTE.2015.2472963

26. Lydia M, Kumar S.S, Selvakumar A.I, Kumar G.E.P. A comprehensive review on wind turbine power curve modeling techniques. Renewable and Sustainable Energy Reviews. 2014; 30: 452-460. doi: 10.1016/j.rser.2013.10.030.

27. Kusiak A, Zheng H, Song Z. Models for monitoring wind farm power. Renewable Energy. 2009; 34(3): 583-590.doi: 10.1016/j.renene.2008.05.032.

28. Pandit R, Infield D. Comparison of binned and Gaussian Process based wind turbine power curve for condition monitoring purposes. Journal of Maintenance Engineering. (accepted/in press), 2017; 2:314-325, University of Manchester, UK. 
https://pure.strath.ac.uk/portal/en/publications/comparison-of-binned-and-gaussian-processbased-wind-turbine-power-curves-for-condition-monitoring-purposes(29dc1a54-117d-4123930f-e7aaf789a736).html.

29. Bulaevskaya V, Wharton S, Clifton A, Qualley G, Miller W.O. Wind power curve modeling in simple and complex terrain using statistical models. Journal of Renewable and Sustainable Energy.2015; 7(1): 013103.doi:10.1063/1.4904430.

30. Schlechtingen M, Santos I. Comparative analysis of neural network and regression based condition monitoring approaches for wind turbine fault detection. Mechanical Systems and Signal Processing.2011; 25(5): 1849-1875.doi: 10.1016/j.ymssp.2010.12.007.

31. Rasmussen C, Williams C. Gaussian Processes for Machine Learning. Publisher: MIT Press. 2005. ISBN 026218253X.

32. Gonzalez J. Linear-Time Inverse Covariance Matrix Estimation in Gaussian Processes. 2017. http://www.cs.cmu.edu/ sahong/docs/jegonzal_sahong_final.pdf. doi: arXiv:1610.08035v4. (accessed $1^{\text {st }}$ March 2018).

33. Hartikainen J, Särkkä S. Kalman filtering and smoothing solutions to temporal Gaussian process regression models. In: Proceedings of IEEE International Workshop on Machine Learning for Signal Processing, Kittila;2010, pp. 379-384. doi: 10.1109/MLSP.2010.5589113.

34. Sarkka S, Solin A, Hartikainen. Spatiotemporal Learning via Infinite-Dimensional Bayesian Filtering and Smoothing: A Look at Gaussian Process Regression Through Kalman Filtering. IEEE Signal Processing Magazine. 2013; 30 (4): 51-61. doi: 10.1109/MSP.2013.2246292.

35. Chen J, Cao N. Parallel Gaussian Process Regression with Low-Rank Covariance Matrix Approximations. In: Proceedings of the Twenty-Ninth Conference on Uncertainty in Artificial Intelligence (UAI2013); 2013, Bellevue, Washington, USA. doi: https://arxiv.org/abs/1408.2060.

36. Li P, Chen S. Gaussian Process Latent Variable Models. CAAI Transactions on Intelligence Technology.2016; 1(4): 366-376. doi: 10.1016/j.trit.2016.11.004.

37. Pandit R, Infield D. SCADA - based wind turbine anomaly detection using Gaussian Process models for wind turbine condition monitoring purposes. IET Renewable Power Generation.2018, Vol 12, issue 11.pp. 1249-1255. doi: $\underline{\text { 10.1049/iet-rpg.2018.0156. }}$ 
38. Neyman J. Outline of a Theory of Statistical Estimation Based on the Classical Theory of Probability. Philosophical Transactions of the Royal Society A.1937; 236: 333-380. doi:10.1098/rsta.1937.0005.

39. Wilk M, Gnanadesikan R. Probability plotting methods for the analysis for the analysis of data. Biometrika.1986; 55(1):1-17.doi: www.jstor.org/stable/2334448.

40. Dickinson J, Chakraborti S. Nonparametric statistical inference. Publisher: CRC Press (4th ed.), ISBN 978-0-8247-4052-8, 2003. 